\title{
Multiplexed Online-Monitoring of Microfluidic Free-Flow Electrophoresis via Mass Spectrometry
}

\author{
Matthias Jender, Pedro Novo, Dominic Maehler, Ute Münchberg, Dirk Janasek, Erik Freier* \\ Institut für Analytische Wissenschaften - ISAS - e.V., Dortmund, Germany \\ *erik.freier@isas.de
}

\section{Contents}

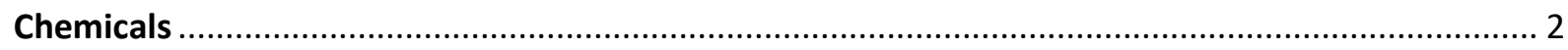

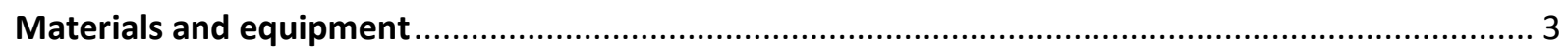

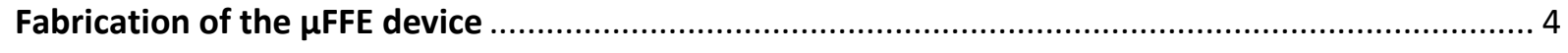

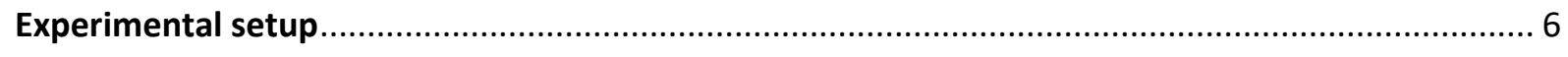

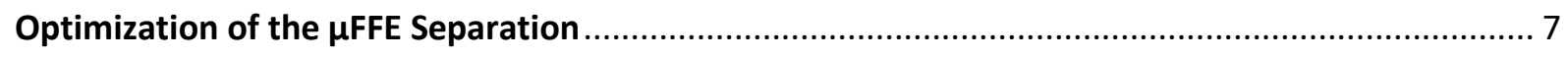

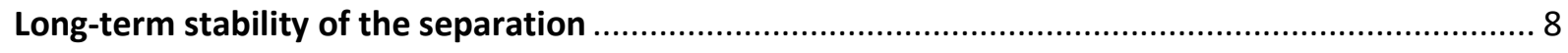

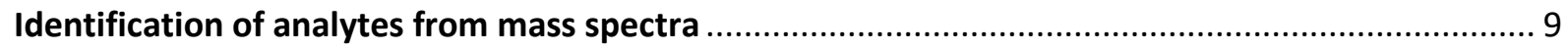




\section{Chemicals}

$\begin{array}{lll}\text { Substance } & \text { Purity } & \text { Source } \\ \text { 2-propanol } & \text { ULC/MS grade } & \text { Biosolve BV, Valkenswaard, NED } \\ \text { Adenosine 5'-monophosphate disodium salt } & \geq 99 \% \text { HPLC grade } & \text { Sigma-Aldrich, Steinheim, GER } \\ \text { Adenosine 5'-diphosphate sodium salt } & \geq 95 \% & \text { Sigma-Aldrich, Steinheim, GER } \\ \text { Adenosine 5'-triphosphate disodium salt hydrate } & \geq 99 \% \text { grade I } & \text { Sigma-Aldrich, Steinheim, GER } \\ \text { Ammonium acetate } & \text { p. a. } & \text { Carl Roth, Karlsruhe, GER } \\ \text { Coenzyme A trilithium salt } & \geq 95 \% & \text { Biomol, Hamburg, GER } \\ \text { Sodium dihydrogen phosphate monohydrate } & \text { p. a. } & \text { Merck, Darmstadt, GER }\end{array}$




\section{Materials and equipment}

\begin{tabular}{|c|c|}
\hline Material / Equipment & Source \\
\hline 3M 9969 Adhesive Transfer Tape & 3M, Saint Paul, USA \\
\hline 3M 9965 Double-Sided Polyester Diagnostic Tape & 3M, Saint Paul, USA \\
\hline Capillary Electrophoresis instrument PA 800 Plus & SCIEX, Darmstadt, GER \\
\hline Cutting plotter Silhouette Cameo 3 & Silhouette America, USA \\
\hline Dosage needles, stainless steel: ID $0.20 \mathrm{~mm}$, OD $0.41 \mathrm{~mm}$ & Vieweg, Kranzberg, GER \\
\hline Dosage needles, stainless steel: ID $0.41 \mathrm{~mm}$, OD $0.71 \mathrm{~mm}$ & Vieweg, Kranzberg, GER \\
\hline Fused silica capillaries: ID $50 \mu \mathrm{m}$, OD $186 \mu \mathrm{m}$ & Chromatographie Handel Müller, Fridolfing, GER \\
\hline Fused silica capillary: ID $50 \mu \mathrm{m}$, OD $360 \mu \mathrm{m}$ & Polymicro Technologies, Phoenix, USA \\
\hline HCN 7 E-350 power supply unit & FuG Elektronik, Schechen, GER \\
\hline Ion Max atmospheric pressure ionization housing & Thermo Fisher Scientific, USA \\
\hline Ismatec peristaltic pump IPC-N ISM939D & Cole-Parmer, USA \\
\hline Ismatec peristaltic pump tubing, SC0188T, Tygon LMT - 55 & Cole-Parmer, USA \\
\hline LTQ Orbitrap Velos Pro & Thermo Fisher Scientific, USA \\
\hline Multimeter DMM220 & Multimetrix, FRA \\
\hline Multimeter 72-1016 & Tenma, USA \\
\hline neMESYS B002-01 & Cetoni, Korbussen, GER \\
\hline Nuclepore track-edged polycarbonate membranes & Whatman, Maidstone, UK \\
\hline PEEK capillary ID $64 \mu \mathrm{m}$, OD $1 / 16 ”$ & Vici International, Schenkon, SUI \\
\hline Platinum wire, $0.5 \mathrm{~mm}$ diam. & Sigma-Aldrich CHEMIE, Steinheim, GER \\
\hline Poly(methyl methacrylate) (PMMA) & Thyssenkrupp Plastics, Essen, GER \\
\hline Polytetrafluoroethylene tubing: ID $0.5 \mathrm{~mm}$, OD $1.6 \mathrm{~mm}$ & Bohlender, Grünsfeld, GER \\
\hline Silicone rubber Elastosil E41 & Wacker Chemie, München, GER \\
\hline Valve Cheminert C5FH-0005 & Vici Valco Instruments, Schenkon, SUI \\
\hline Water purification unit: PURELAB flex & ELGA LabWater, Lane End, UK \\
\hline
\end{tabular}




\section{Fabrication of the $\mu$ FFE device}

The microfluidic device used in this work is a novel device based on a previous design ${ }^{1}$. With the exception of the porous membranes used, the materials employed were changed. The current device is mechanically stable, can be stored easily, is simple to fabricate and reliable (the device can be used and reused over a period of more than 12 weeks). The following description of the microfluidic device is accompanied by an exploded view (Figure S1), which is also presented in smaller scale as Figure 2.

The microstructures in the chip are formed using double-sided, medical grade polyester adhesive tape (9965, 3M) with a thickness of approximately $90 \mu \mathrm{m}(\mathrm{d})^{2}$. The tape consists of a polyester carrier $(50 \mu \mathrm{m})$, acrylate adhesive on both sides and silicone coated polyester release liners covering the adhesive surfaces. The $2 \mathrm{D}$ microfluidic channel shape is designed in a CAD software and transferred to the adhesive tape using a common all-purpose cutting plotter (Cameo 3, Silhouette America). After cutting the microfluidic structures into the tape, the excess pieces of tape (corresponding to the shape of separation bed, inlet and outlet channels) are discarded. Then, the release liner is removed from one side of the tape, exposing the adhesive. A $2 \mathrm{~mm}$ thick PMMA plate (a) is carefully placed onto the adhesive aligning the equal rectangular shapes of plate and tape. The adhesive on the other side of the tape is then exposed by removing the second release liner. Afterwards, the top layer (b), another $2 \mathrm{~mm}$ thick PMMA plate, is carefully placed onto the exposed adhesive surface. The top layer is custom designed and fabricated in-house with access holes as inlets, outlets and vias (vertical electrical connections) (e-g) to the reservoirs (c). Special care is taken to precisely align the holes in the top plate with the microfluidic structures of the adhesive tape. Sandwiched between the PMMA plates, the tape's thickness (carrier + adhesive) defines the height of the microfluidic structures (approx. $90 \mu \mathrm{m}$ ). To ensure a comprehensive bonding between adhesive tape and PMMA plates, pressure is applied onto the PMMA plates. A thorough bonding can be controlled by eye, since bonded and unbonded areas can be easily distinguished.

The top plate contains 5 inlets $(0.7 \mathrm{~mm}$ diam. $)$, 9 outlets $(0.4 \mathrm{~mm}$ diam. $)$ and 34 vias $(1 \mathrm{~mm}$ diam.). The vias are positioned on either side of the microstructure connecting the electrode reservoirs and the separation chamber. Polycarbonate membranes $(\mathrm{h})$ with $0.1 \mu \mathrm{m}$ pore size (Track-Etched Nuclepore, Whatman) are installed to minimize fluid flow between the microfluidic structures and the electrolyte reservoirs. To install the membranes an adhesive film is applied via adhesive transfer tape (9969, $3 \mathrm{M}$ ) on top of the PMMA plate around the vias. Then, the excess adhesive covering the holes is removed manually using a pin. Next, a piece of membrane, trimmed with scissors, is glued with the adhesive film to cover the holes. As demonstrated in our previous work, this construct prevents hydrodynamic flow between the electrode reservoirs and the separation chamber, while not contributing to a significant increase in electrical resistance ${ }^{1}$. The electric field through the separation chamber is generated between two electrodes (i) made of platinum wire $(0.5 \mathrm{~mm}$ diam.), which are inserted manually into the electrode reservoirs through a $1 \mathrm{~mm}$ diameter hole. The electrodes are guided through another $1 \mathrm{~mm}$ diameter hole at the opposite end of the reservoirs to the electrode interface where they are connected to an external power supply. Silicone rubber (Elastosil E41, Wacker) is applied at the holes to hold the wires in position and to seal the reservoirs. The electrode reservoirs are then glued with adhesive transfer tape $(9969,3 \mathrm{M})$ on top of and in alignment with the membrane-covered holes.

1. Novo, P.; Dell'Aica, M.; Jender, M.; Höving, S.; Zahedi, R. P.; Janasek, D., Integration of polycarbonate membranes in microfluidic free-flow electrophoresis. Analyst 2017, 142 (22), 4228-4239.

2. https://www.3m.com/3M/en_US/company-us/all-3m-products/ /3M-Microfluidic-Diagnostic-Tape-9965-DoubleSided-White-Tape-CONFIGURABLE/?N=5002385+3294739673\&rt=rud [accessed March 26, 2020] 


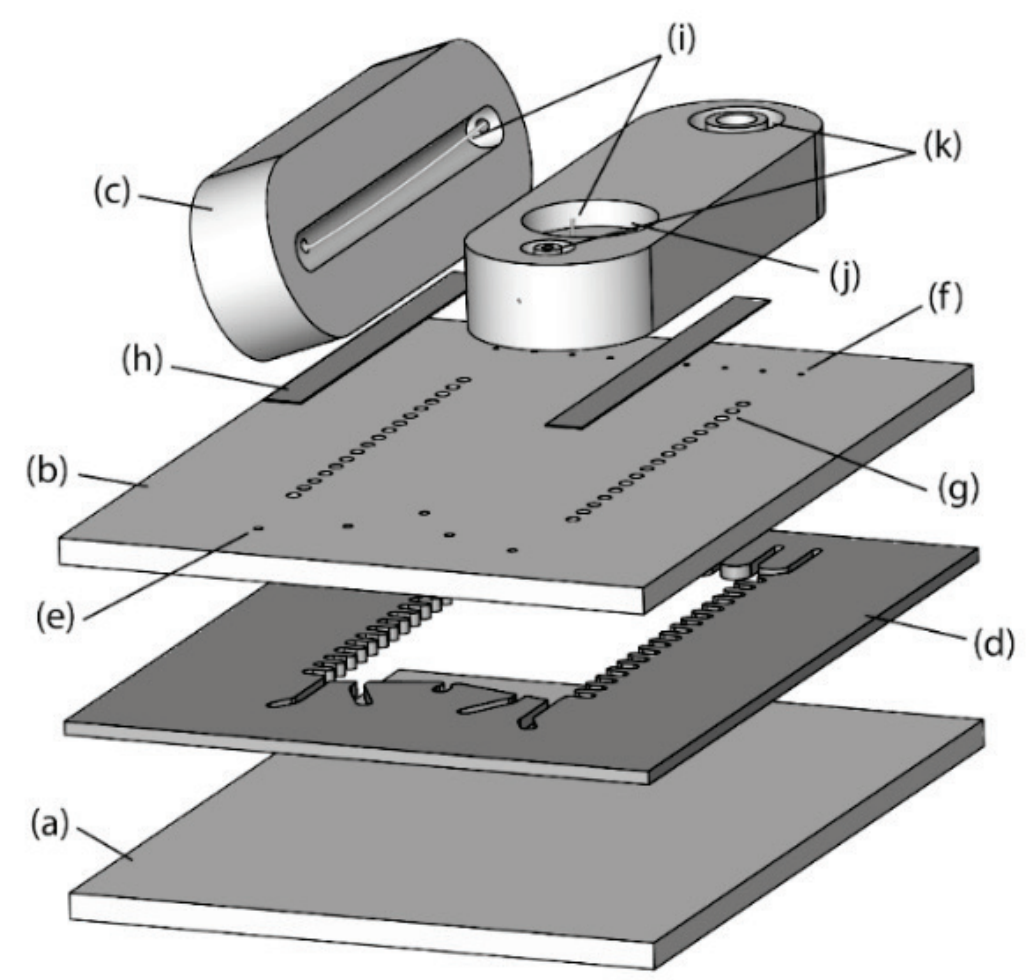

Figure S1. Exploded view of the $\mu$ FFE device. Bottom plate (a), top plate (b), electrode reservoirs (c), microfluidic channels in doublesided adhesive tape (d). The top plate contains inlet holes (e), outlet holes (f) and vias (g), which are covered by polycarbonate membranes (h). Above, the electrode reservoirs are located (c). The left reservoir is rotated in the illustration for a better view of its bottom and the embedded platinum electrode (i). The electrodes can be contacted at the respective interface $(j)$ at the electrode reservoir. Electrolyte solution is supplied via the fluid ports $(\mathrm{k})$. 


\section{Experimental setup}

The experimental setup is shown in the photograph below (Figure S2) and referenced in the following description by the letters from the photograph. Sample and part of the electrolyte solution are injected into the $\mu$ FFE device (a) with a syringe pump (neMESYS B002-01, Cetoni) (b) and polytetrafluoroethylene (PTFE) tubing. Additional electrolyte solution is drawn into the device from electrolyte reservoirs (c) actuated by the peristaltic pump (IPC-N ISM939D, Ismatec) (d), which is connected to the $\mu$ FFE outlets causing negative pressure in the separation chamber. At the inlets the PTFE tubes are plugged onto blunt stainless steel needles $(0.41 \mathrm{~mm} \mathrm{ID}, 0.71 \mathrm{~mm} \mathrm{OD})$, which interface the microchip through holes drilled into the PMMA (Figure S1e). Dead-end PTFE tubes close unused inlets and outlets. The device's outlets are interfaced via stainless steel needles $(0.20 \mathrm{~mm}$ ID, $0.41 \mathrm{~mm}$ OD), which are directly connected to the peristaltic pump tubing ( $0.13 \mathrm{~mm}$ ID, Tygon) by slipping the tubes over the needles. The peristaltic pump tubes are connected on the other side via fused silica (FS) capillaries ( $50 \mu \mathrm{m} \mathrm{ID,} 186 \mu \mathrm{m}$ OD) to the switching valve (Cheminert C5FH-0005, Vici Valco Instruments) (e), which can be operated with the controller (f). The FS capillaries are plugged directly into the peristaltic pump tubes. From the valve's common outlet a PEEK capillary (64 $\mu \mathrm{m}$ ID, $1 / 16$ " OD) leads to the grounding union of the atmospheric pressure ionization (API) housing (g) of the MS (LTQ Orbitrap Velos Pro, Thermo Fisher Scientific). From the other end of the grounding union the FS emitter capillary (50 $\mu \mathrm{m}$ ID, $186 \mu \mathrm{m}$ OD) leads into the API housing where an electric potential difference is applied between the emitter capillary and the MS counter electrode. The syringe pump supplies sheath liquid via the inlet at the ESI probe $(\mathrm{h})$. The electric field in the $\mu$ FFE device is controlled by the power supply (HCN 7 E-350, FuG Elektronik) (i); voltage and current are monitored with multimeters (j).

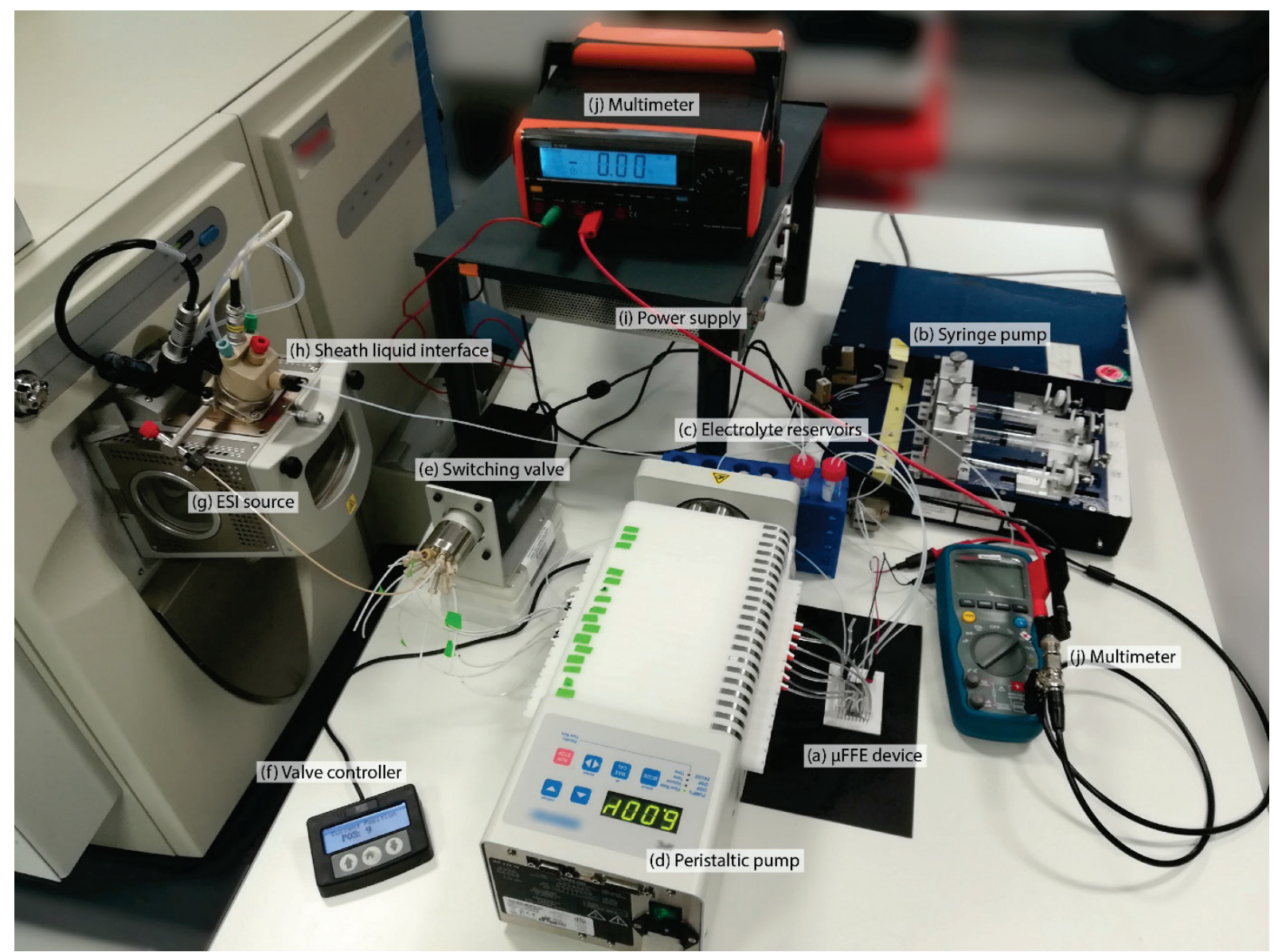

Figure S2. Photograph of the experimental setup for the coupling of $\mu \mathrm{FFE}$ with MS. The shown components are referenced in the text above. For a schematic overview of the experimental setup please refer to Figure 1 in the main text. 


\section{Optimization of the $\mu \mathrm{FFE}$ separation}

The MS-compatible $\mu$ FFE system was established with fluorescent dyes before the coupling to MS. The challenge was to find an electrolyte system (electrolyte type and concentration for both separation bed and the electrolyte reservoirs) that yielded good electrophoretic separation power, was stable over time and compatible to ESI-MS. With the presented setup of $\mu$ FFE coupled to MS, we then optimized the separation conditions (voltage and flow rates) for the analytes AMP, ATP and CoA. We started by letting all analytes exit outlet \#1 without a separation voltage applied (compare to the first segment of Fig. 3 or S3). Then we applied a small voltage and slowly increased its strength until analytes started to disappear from the spectra, thus they migrated in the electric field to another $\mu \mathrm{FFE}$ outlet. We switched to the next outlet channels one by one to see how far the analytes migrated. We successively increased the voltage (or reduced the flow rates) until the analyte with the highest electrophoretic mobility (here ATP) reached the farthest outlet (here outlet \#5) to achieve maximum separation power. Then, voltage or flow rates were adjusted in smaller steps to focus each analyte to a separate outlet. 


\section{Long-term stability of the separation}

To show the stability of the FFE separation over time, the outlet channels were measured a second time in reverse order. The extracted ion chromatogram below (Figure S3) is an extension of the measurement presented in the main paper, which only showed the first 12 minutes (Figure 3). The repeated analysis of the outlet channels shows identical results $(t=12: 00-26: 00$ min) confirming a stable separation.

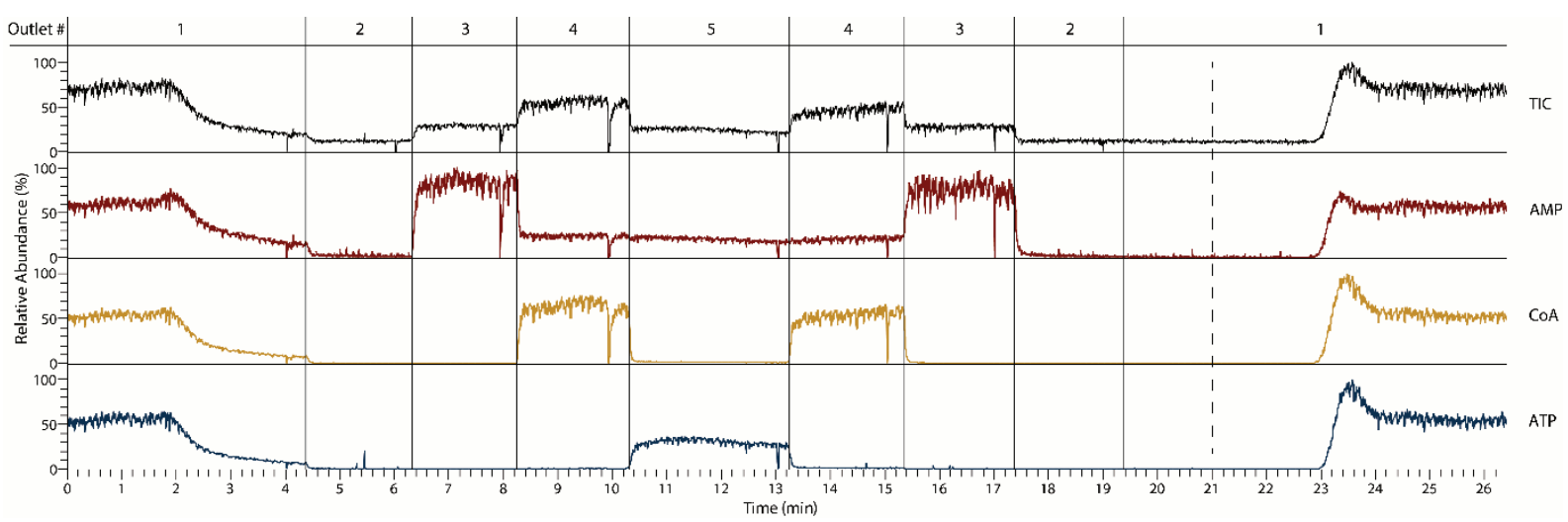

Figure S3. Extracted ion chromatogram of the analytes separated by $\mu$ FFE. The chromatogram is divided into segments by vertical lines. The number of the outlet channel being analyzed at a time by MS is displayed on top of each segment. Voltage was turned on at $t=0$ ' and turned off at $\mathrm{t}=21^{\prime}$ (dashed line). 


\section{Identification of analytes from mass spectra}

Mass spectra taken from each channel during the experiment are shown in Figure S4. The mass range for the measurements was set to $\mathrm{m} / \mathrm{z}=300-850$. The $\mathrm{m} / \mathrm{z}$ assigned to the respective analytes are color-coded (AMP $=$ red, CoA $=$ yellow, ATP $=$ blue). A list of the most abundant $\mathrm{m} / \mathrm{z}$ values and the assigned substances, adducts and fragments is provided in Table S3. The assigned $\mathrm{m} / \mathrm{z}$ values were incorporated in the EIC (Figure S3) with a mass accuracy of $5 \mathrm{ppm}$.

Table S1 List of the mass-to-charge ratios found in the spectra that were assigned to the analytes AMP, ATP and CoA. The fragment phosphopantetheine is abbreviated as PP.

\begin{tabular}{|c|c|c|c|}
\hline Calculated mass & AMP & ATP & CoA \\
\hline 382.5503 & & & {$[\mathrm{M}-2 \mathrm{H}]^{2-}$} \\
\hline 505.9885 & & {$[\mathrm{M}-\mathrm{H}]^{-}$} & \\
\hline 527.9710 & & {$[\mathrm{M}-2 \mathrm{H}+\mathrm{Na}]^{-}$} & \\
\hline 549.9535 & & {$[\mathrm{M}-3 \mathrm{H}+2 \mathrm{Na}]^{-}$} & \\
\hline 788.0904 & & & {$[\mathrm{M}-2 \mathrm{H}+\mathrm{Na}]^{-}$} \\
\hline 810.0729 & & & {$[\mathrm{M}-3 \mathrm{H}+2 \mathrm{Na}]^{-}$} \\
\hline
\end{tabular}




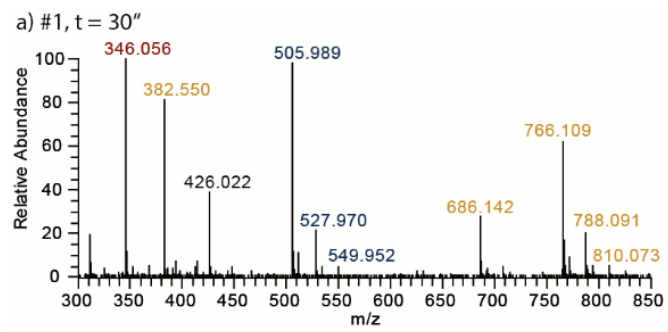

b) $\# 2, \mathrm{t}=5^{\prime}$
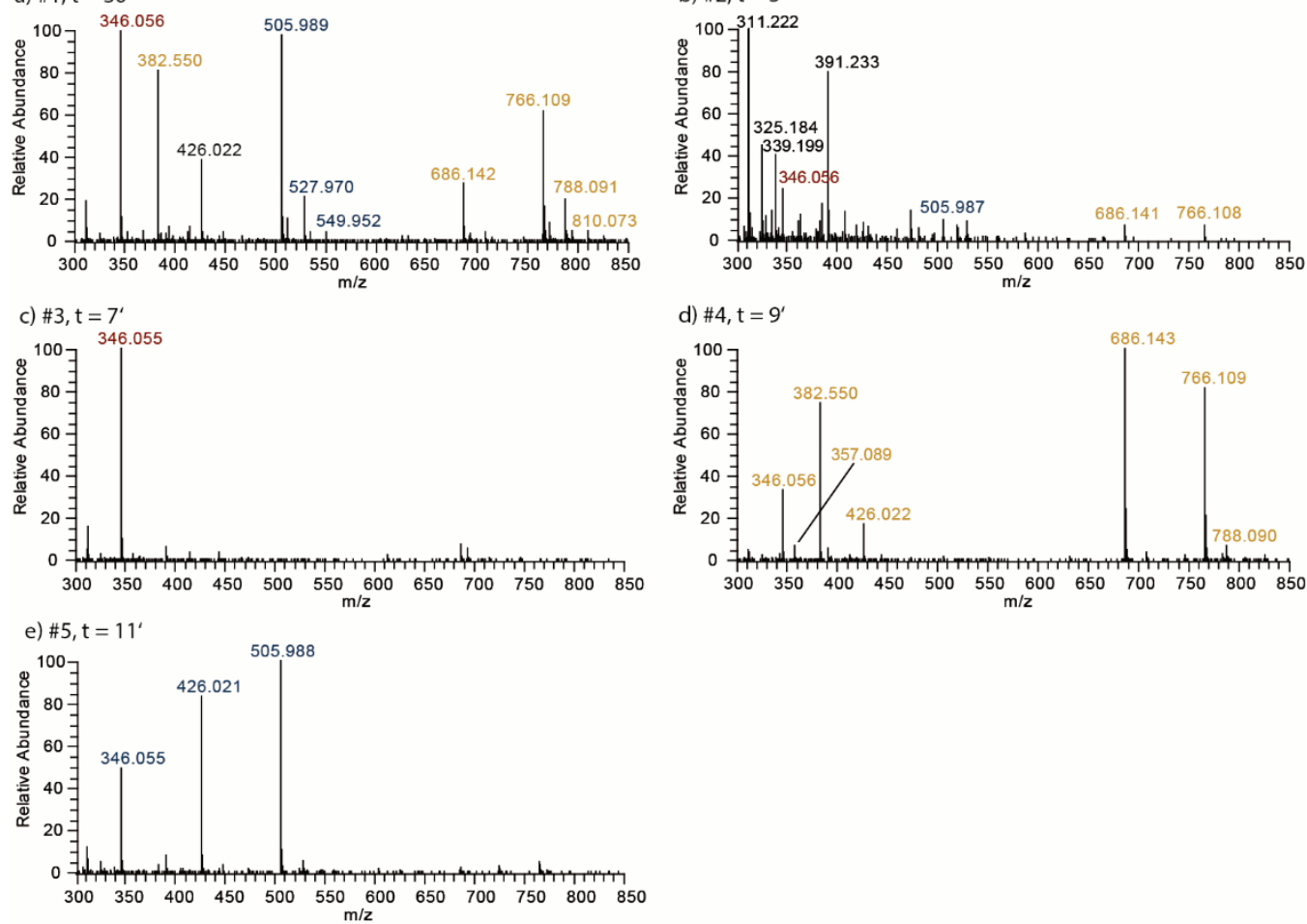

Figure S4. Mass spectra of each of the five $\mu \mathrm{FFE}$ outlet channels. The respective $\mu \mathrm{FFE}$ outlet channel number and the time point are displayed on top of each mass spectrum. The $\mathrm{m} / \mathrm{z}$ assigned to the analytes are color-coded: $\mathrm{AMP}=$ red, CoA $=$ yellow, ATP = blue. ( $\mathrm{a}$ ) Absent of an electric field all analytes are present. (b) Low signal intensity. Only traces of the analytes are detected besides some unassigned peaks. (c) AMP is predominant. (d) CoA shows several fragments, adducts and different charge states (see Table S3). (e) Peaks for ATP and its dephosphorylated fragments AMP and ADP are predominant. 